\title{
Immunogenicity Specimen Assessments Original Result Unit
}

National Cancer Institute

\section{Source}

National Cancer Institute. Immunogenicity Specimen Assessments Original Result Unit. NCl Thesaurus. Code C117560.

The unit of measure for the result of the immunogenicity specimen assessment as

originally received or collected. 